\title{
Unified Approach for Energy Detection of Unknown Deterministic Signal in Cognitive Radio over Fading Channels
}

\author{
Sanjeewa P. Herath*, Nandana Rajatheva ${ }^{\dagger}$ \\ Telecommunications Field of Study, \\ Asian Institute of Technology, \\ Thailand. \\ Email: sanjeewa@ieee.org*, rajath@ait.ac.th ${ }^{\dagger}$
}

\author{
Chintha Tellambura \\ Department of Electrical alnd Computer Engineering, \\ University of Alberta, \\ Canada. \\ Email: chintha@ece.ualberta.ca
}

\begin{abstract}
Detection of an unknown deterministic signal by using an energy detector is of promising for cognitive radio networks. In this paper, a new approach is proposed to analyze the performance of the energy detector. It is based on the contour integral representation of the Marcum- $Q$ function and the use of the moment generating function (MGF) of the signal-to-noise ratio (SNR). A new decision variable is constructed for the case of maximal ratio combining (MRC) reception. With its help and the MGF based approach, the performance of the MRC energy detector over i.i.d. Rician fading channels is analyzed. This case is intractable with the conventional probability density function (PDF) based approach. Further the detection probability of MRC combined energy detector over Nakagami- $m$ fading branches is derived. The simulation results are presented to support the developed MGF based method, decision variable formulation and derivations. The detector performance is evaluated over different fading and diversity parameters with the help of numerical and simulation examples.
\end{abstract}

\section{INTRODUCTION}

Cognitive radio technology allows unlicensed users (secondary users) to dynamically use unoccupied free spectrum bands of primary users (licensed users), while avoiding interference to primary users. Energy detection can be used to explore the presence of primary user transmissions and hence to identify the available spectrum holes. When using this technique, the energy detector of the secondary user treats the received primary user transmission as an unknown deterministic signal. Hence, secondary users do not requires unauthorized, irrelevant details of the primary transmissions. Due to this application, the performance of the energy detector using diversity reception techniques and over various wireless fading environments is of interest.

The technique of detecting an unknown deterministic signal by using an energy detector is introduced in [1]. It is shown that the detection problem is a test of binary hypotheses and statistics of the decision variable is chi-square distributed, irrespective of whether the process model is lowpass or bandpass.

Kostylev [2] extends the formulation to fading channels. In his work, average detection $\left(P_{d}\right)$ and false alarm $\left(P_{f}\right)$ probabilities over Rayleigh, Rician and Nakagami- $m$ fading channels are presented. But the Nakagami- $m$ channel result is limited to an integral form expression. In [3] Nakagami- $m$ and Rician fading channels are considered. But the derivation of Nakagami- $m$ is restricted to integer values of the shape parameter $(m)$ while the result of Rician fading channel is limited to unity time bandwidth product $(u)$ in the decision variable.

Maximal ratio, selection and switch and stay diversity detectors are analyzed over i.i.d. Rayleigh fading channels in [3]. Expressions of $P_{d}$ over i.i.d. and correlated Rayleigh fading channels with square-law combining is derived in [4] while square-law selection combining is considered over i.i.d. Rayleigh fading branches. Selection and maximal ratio combining are analyzed in [5] over Rayleigh fading channels.

Careful study of prior work reveals that the performance analysis over diversity detectors is limited to Rayleigh fading channel. This is mainly due to the lack of integrals involving Marcum-Q function and the use of limited results available in [6] and [7]. Further, none of these works provide simulation results to support their theoretical formulations. In our work presented in [8], an alternative infinite series form expression for $P_{d}$ over Nakagami- $m$ fading channel is obtained. Further, performance of the equal gain diversity combined energy detector is analyzed over i.i.d. Nakagami- $m$ channels. In addition, we derive exact expressions for $P_{d}$ and $P_{f}$ over square-law and switch and stay diversity detectors in [9]. All these analyses (previous and our work) follow the PDF based approach. In other words, after finding the conditional probability the corresponding expression is integrated over the PDF of output signal-to-noise ratio (SNR). This conditional detection probability is of the form of Marcum-Q function and thus the resulting integrals are often difficult to evaluate in general. In this paper, we use the MGF based approach and an alternative contour integral representation of MarcumQ to transform the integral to complex domain. This process avoids many difficulties faced in evaluating integrals involving Marcum-Q and yet gives simpler closed form results. Applying this method, Nakagami- $m$ and Rician fading channels are analyzed. A new decision variable is formed over a maximal ratio combiner. Taking the branch statistics to be i.i.d. Nakagami- 
$m$ and Rician fading, exact expressions of $P_{d}$ are derived. It should be noted that the Rician fading channel is intractable in general with the PDF based approach.

The organization of the rest of the paper is as follows. In Section II, we formulate the decision variable over a fading channel. Section III and IV present the derivation of $P_{d}$ over Nakagami- $m$ and Rician fading channels respectively. In Section V analysis over a maximal ratio combiner is provided. Section VI presents numerical and simulation results and the concluding remarks are given in Section VII.

\section{System ModeL}

To be consistent, notations similar to [8] are used as listed below.

$N_{01} \quad$ : One sided noise power spectral density

$W \quad$ : One sided bandwidth

$T \quad$ : Observation time interval

$u=T W$ : Time bandwidth product

$\chi_{2 u}^{2} \quad$ : Central Chi-square distribution with $2 u$ degree of freedom

$\chi_{2 u}^{2}(\epsilon)$ : Non central Chi-square distribution with $2 u$ degree of freedom and non centrality parameter $\epsilon$

$E_{s} \quad$ : Observed energy

The detection of the existence of the unknown deterministic signal $s(t)$, is a binary hypothesis test as shown in (1),

$$
y(t)= \begin{cases}n(t) & : H_{0} \\ h s(t)+n(t) & : H_{1}\end{cases}
$$

where $n(t)$ is white Gaussian noise and $h$ is the channel coefficient amplitude [1]. Hence a sample from noise process $n_{i}$ is a Gaussian random variable with zero mean and $N_{01} W$ variance; $n_{i} \sim N\left(0, N_{01} W\right)$ [1]. The energy detector decision variable $(Y)$ is constructed as in (2),[1].

$$
Y=\frac{2}{N_{01}} \int_{0}^{T} n^{2}(t) d t=\sum_{i=1}^{2 u}\left(\frac{n_{i}}{\sqrt{N_{01} W}}\right)^{2} \quad: H_{0}
$$

Thus, $Y$ under $H_{0}$ is a square sum of $2 u$ Gaussian random variables of $N(0,1)$ and its statistics follows $\chi_{2 u}^{2}$. Similarly, $Y$ under $H_{1}$ is formed as given by (3).

$$
Y=\frac{2}{N_{01}} \int_{0}^{T} y^{2}(t) d t=\sum_{i=1}^{2 u}\left(\frac{h s_{i}+n_{i}}{\sqrt{N_{01} W}}\right)^{2} \quad: H_{1}
$$

Here, we assume that $h$ is constant over the $2 u$ samples. It therefore follows that, $Y$ under $H_{1}$ is $\chi_{2 u}^{2}(\epsilon)$ where $\epsilon$ is given by (4), [1].

$$
\epsilon=\sum_{i=1}^{2 u}\left(\frac{h s_{i}}{\sqrt{N_{01} W}}\right)^{2}=\frac{h^{2}}{W N_{01}} \sum_{i=1}^{2 u} s_{i}^{2}=\frac{2 h^{2} E_{s}}{N_{01}}=2 \gamma
$$

Here, SNR is defined by $\gamma=\frac{h^{2} E_{s}}{N_{01}}$.

Thus, the probability of detection $\left(P_{d}\right)$ and probability of false alarm $\left(P_{f}\right)$ can be written as in (5) and (6) respectively where $\lambda$ is the threshold of the detector [4].

$$
P_{d}=Q_{u}(\sqrt{2 \gamma}, \sqrt{\lambda})
$$

$$
P_{f}=\frac{\Gamma\left(u, \frac{\lambda}{2}\right)}{\Gamma(u)}
$$

Here $Q_{u}(.,$.$) is the generalized ( u^{\text {th }}$ order) Marcum Qfunction and $\Gamma(.,$.$) is upper incomplete gamma function$ which is defined by the integral form $\Gamma(a, x)=\int_{x}^{\infty} t^{a-1} e^{-t} d t$ and $\Gamma(a, 0)=\Gamma(a)$.

Using the contour integral representation of generalized Marcum Q-function given in [10], (5) can be written as in (7), where $\Delta$ is a circular contour of radius $r$ that encloses the origin $(0<r<1)$.

$$
P_{d}=\frac{e^{-\frac{\lambda}{2}}}{2 \pi j} \oint_{\Delta} \frac{e^{\left(\left(\frac{1}{z}-1\right) \gamma+\frac{\lambda}{2} z\right)}}{z^{u}(1-z)} d z
$$

The MGF of $\gamma$ is $M(s)=E\left(e^{-s \gamma}\right)$ where $E($.$) represents$ mathematical expectation. Thus, the average detection probability $\left(\bar{P}_{d}\right)$ can be expressed as,

$$
\bar{P}_{d}=\frac{e^{-\frac{\lambda}{2}}}{2 \pi j} \oint_{\Delta} M\left(1-\frac{1}{z}\right) \frac{e^{\frac{\lambda}{2} z}}{z^{u}(1-z)} d z .
$$

\section{Detection over Nakagami- $m$ Fading Channel}

The MGF of SNR of Nakagami- $m$ fading $M_{\gamma_{N a k}}(s)$ is,

$$
M_{\gamma_{N a k}}(s)=\frac{1}{\left(1+\frac{\bar{\gamma} s}{m}\right)^{m}}, \quad m \geq \frac{1}{2}
$$

where $\bar{\gamma}$ is average SNR and $m$ is Nakagami- $m$ distribution shape parameter. Thus using (8), average detection probability over Nakagami- $m$ fading $\left(\bar{P}_{d, N a k}\right)$ can be written as,

$$
\bar{P}_{d, N a k}=\left(\frac{m}{m+\bar{\gamma}}\right)^{m} e^{-\frac{\lambda}{2}} \frac{1}{2 \pi j} \oint_{\Delta} f(z) d z,
$$

where $f(z)$ is given in (11) and $\theta=\frac{\bar{\gamma}}{m+\bar{\gamma}}$.

$$
f(z)=\frac{e^{\frac{\lambda}{2} z}}{(z-\theta)^{m} z^{u-m}(1-z)}
$$

The contour integral in (10) is evaluated for integer $m$ as shown in subsections below.

Case I: $u>m$

The integral in (10) contains $m$ and $u-m$ order poles at $\theta$ and origin respectively. From the residue theorem, $\bar{P}_{d, N a k}$ can be derived as in (12), where $\operatorname{Res}\left(f ; z_{0}\right)$ denotes the residue of function $f(z)$ at $z_{0}$.

$$
\bar{P}_{d, N a k}=\operatorname{Res}(f ; \theta)+\operatorname{Res}(f ; 0)
$$

The Res $(f ; \theta)$ and $\operatorname{Res}(f ; 0)$ for Nakagami- $m$ fading channel are given in (13) and (14) respectively.

$$
\begin{gathered}
\operatorname{Res}(f ; \theta)=\lim _{z \rightarrow \theta} \frac{1}{(m-1) !} \frac{d^{m-1}}{d z^{m-1}}\left(\frac{e^{\frac{\lambda}{2} z}}{z^{u-m}(1-z)}\right) \\
\operatorname{Res}(f ; 0)=\lim _{z \rightarrow 0} \frac{1}{(u-m-1) !} \frac{d^{u-m-1}}{d z^{u-m-1}}\left(\frac{e^{\frac{\lambda}{2} z}}{(z-\theta)^{m}(1-z)}\right)
\end{gathered}
$$




\section{Case II: $u \leq m$}

Under this condition, no poles exist at the origin. Thus,

$$
\bar{P}_{d, N a k}=\operatorname{Res}(f ; \theta) .
$$

As a by product, average detection probability over Rayleigh fading channel $\left(\bar{P}_{d, \text { Ray }}\right)$ can be obtained by substituting $m=1$ in (12) and (15) and shown in (18) (bottom of this page). The evaluation of derivatives and limits in (13) and (14) yields simpler expressions over Nakagami- $m$ and Rayleigh fading channels. These results are numerically equivalent to the respective results in [3]-[5] and [8].

\section{Detection over Rician Fading Channel}

The MGF of SNR of Rician fading $M_{\gamma_{R i c}}(s)$ is,

$$
M_{\gamma_{R i c}}(s)=\frac{1+K}{(1+k+s \bar{\gamma})} \exp \left[-\frac{s K \bar{\gamma}}{(1+K+s \bar{\gamma})}\right]
$$

where $K$ is the Rice factor. Hence, using (8), average detection probability over Rician fading $\left(\bar{P}_{d, R i c}\right)$ can be written as,

$$
\bar{P}_{d, R i c}=\frac{(1+K) \theta e^{-\left(\frac{\lambda}{2}+K \theta\right)}}{2 \pi j \bar{\gamma}} \oint_{\Delta} \frac{e^{\left(\frac{a}{z-\theta}+\frac{\lambda z}{2}\right)}}{(z-\theta) z^{u-1}(1-z)} d z
$$

where $\theta=\frac{\bar{\gamma}}{\bar{\gamma}+K+1}$ and $a=K \theta(1-\theta)$. For the special case of $K=0$ (Rayleigh fading), (19) reduces to Rayleigh equivalent form in (10). Applying Laurent series expansion for $\frac{\exp \left(\frac{a}{z-\theta}\right)}{(z-\theta)}$ when $K \neq 0$ and using Residue theorem to integrate term by term, $\bar{P}_{d, R i c}$ for $u>1$ can be expressed as in (20) (bottom of this page). When $u=1$, the pole at 0 disappears. Hence the result can be obtained by setting the limit value of first derivative in (20) to 0 .

\section{Detection with Maximal Ratio Combining}

$L$ branch MRC output under $H_{1}$ can be expressed as given in (21),

$$
r(t)=g s(t)+n(t)
$$

where $g=\sum_{l=1}^{L} h_{l}^{2}$ and $n(t)=\sum_{l=1}^{L} h_{l} n_{l}(t) e^{-j \phi}$. Here $n_{l}(t)$ is a random process with $N\left(0, N_{01} W\right)$ and $h_{l}$ is the channel coefficient. Hence $n(t)$ is a random process with
$N\left(0, \sum_{l=1}^{L} h_{l}^{2} N_{01} W\right)$. Thus, $Y_{m r}$ defined by (22) follows $\chi_{2 u}^{2}$ under $H_{0}$.

$$
Y_{m r}=\frac{2}{g N_{01}} \int_{0}^{T} n^{2}(t) d t=\sum_{i=1}^{2 u}\left(\frac{n_{i}}{\sqrt{g N_{01} W}}\right)^{2}: H_{0}
$$

Hence, average false alarm probability over a maximal ratio combiner is the same given in (6).

Similarly, $Y_{m r}$ under $H_{1}$ is given in (23) and follows $\chi_{2 u}^{2}(\epsilon)$ where $\epsilon$ is given by (24).

$$
\begin{aligned}
& Y_{m r}=\frac{2}{g N_{01}} \int_{0}^{T} r^{2}(t) d t=\sum_{i=1}^{2 u}\left(\frac{g s_{i}+n_{i}}{\sqrt{g N_{01} W}}\right)^{2}: H_{1} \\
& \epsilon=\sum_{i=1}^{2 u}\left(\frac{g s_{i}}{\sqrt{g N_{01} W}}\right)^{2}=\frac{g}{N_{01} W} \sum_{i=1}^{2 u} s_{i}^{2}=\frac{2 g E_{s}}{N_{01}}=2 \gamma_{m r}
\end{aligned}
$$

Here $\gamma_{m r}$ is defined as $\gamma_{m r}=\frac{g E_{s}}{N_{01}}$, [11, eqn.6.22, pp.282].

The statistics of the decision variable of a MRC combiner is claimed to follow $\chi_{2 L u}^{2}\left(\gamma_{m r}\right)$ [5, Section III (A)] but a detailed formulation is not presented. The statistics of the decision variable presented in this paper is different to what is given in [5] and hence the results are also different.

\section{A. Nakagami-m Fading}

The output SNR of $L$ branch MRC combiner $\left(\gamma_{m r}\right)$ is $\gamma_{m r}=\sum_{i=1}^{L} \gamma_{i}$ where $\gamma_{i}$ is the $i^{t h}$ indexed branch SNR. Thus, for i.i.d. branch statistics, the MGF of output SNR $M_{\gamma_{m r, N a k}}(s)$ is given by (25).

$$
M_{\gamma_{m r, N a k}}(s)=\frac{1}{\left(1+\frac{\bar{\gamma} s}{m}\right)^{L m}}, \quad m \geq \frac{1}{2}
$$

The average detection probability over MRC combiner over Nakagami- $m$ channel $\left(\bar{P}_{d, m r, N a k}\right)$ is,

$$
\bar{P}_{d, m r, N a k}=\left(\frac{m}{m+\bar{\gamma}}\right)^{L m} e^{-\frac{\lambda}{2}} \frac{1}{2 \pi j} \oint_{\Delta} f(z) d z,
$$

where $f(z)$ is given by (11) with $m$ replaced by $L m$. Following a similar line of arguments as in Section (III), $\bar{P}_{d, m r, N a k}$ can be expressed in closed form for integer values of $L m$. For the cases $u>L m$ and $u \leq L m$, integral in (26) can be

$$
\bar{P}_{d, \text { Ray }}= \begin{cases}e^{-\frac{\lambda}{2(1+\bar{\gamma})}} & \text { for } u=1 \\ \left(\frac{e^{-\frac{\lambda}{2}}}{1+\bar{\gamma}}\right)\left(\frac{e^{\frac{\lambda \theta}{2}}}{\theta^{u-1}(1-\theta)}+\frac{1}{(u-2) !} \lim _{z \rightarrow 0} \frac{d^{u-2}}{d z^{u-2}}\left[\frac{e^{\frac{\lambda}{2} z}}{(z-\theta)(1-z)}\right]\right) & \text { for } u>1\end{cases}
$$

$$
\begin{array}{r}
\bar{P}_{d, R i c}=\frac{(1+K) \theta e^{-\left(\frac{\lambda}{2}+K \theta\right)}}{\bar{\gamma}} \sum_{n=1}^{\infty} \frac{a^{n-1}}{(n-1) !}\left(\frac{1}{(u-2) !} \lim _{z \rightarrow 0} \frac{d^{u-2}}{d z^{u-2}}\left[\frac{e^{\frac{\lambda z}{2}}}{(1-z)(z-\theta)^{n}}\right]\right. \\
\left.+\frac{1}{(n-1) !} \lim _{z \rightarrow \theta} \frac{d^{n-1}}{d z^{n-1}}\left[\frac{e^{\frac{\lambda z}{2}}}{z^{u-1}(1-z)}\right]\right)
\end{array}
$$


evaluated similar to (12) and (15) respectively. In computing the residues at $\theta$ and $0, m$ should be replaced by $L m$ in (13) and (14).

\section{B. Rician Fading}

Following a similar procedure as in subsection (V-A) and by means of (17), the MGF of the output SNR of MRC receiver over i.i.d. Rician fading channel can easily be found. After substituting this MGF in (8), we arrive at an integral similar to (19). By following same line of arguments as in Section (IV), detection probability over a MRC combined Rician fading branches $\left(\bar{P}_{d, m r, R i c}\right)$ can be derived as in (27) for $u>L$ where $\theta=\frac{\bar{\gamma}}{\bar{\gamma}+K+1}$ and $a=K \theta L(1-\theta)$. When $u \leq L$, the pole at 0 disappears and thus the result can be obtained by setting the limit value of first derivative in (27) to 0 . It is easy verify that when $L=1,(27)$ reduces to (20).

\section{Numerical AND Simulation Results}

The performance of the energy detector is analyzed by means of average $P_{d}$ vs SNR curves for a fixed value of $P_{f}$. First, the energy threshold $(\lambda)$ for the specified $P_{f}$ is evaluated from (6) and using that $\lambda$, average $P_{d}$ is computed. MATHEMATICA is used for computational purposes.

The simulation results are obtained by modeling the wireless channel and constructing the decision variable as described in each case (e.g., $Y_{m r}$ in MRC). Comparing the computed value of decision variable and $\lambda$ (which corresponds to the specified $P_{f}$ ), the decision is taken. By repeating this procedure a sufficiently large number of times, $P_{d}$ and $P_{f}$ values are simulated. It is found that $10^{6}$ repeated transmissions fit the simulation result with the analytical expression figures. Selected results of $P_{d}$ are shown. The results of $P_{f}$ are within the range specified for that simulation. Fig.1 and Fig.2 illustrate the energy detector performance variation over the $m$ and $K$ values respectively. Higher values of $m$ and $K$ show better performance but at low $\bar{\gamma}$ region $(0 d B \sim 5 d B)$, higher $m$ and $K$ values do not give better performance. Thus we conclude that SNR dominates over $m$ and $K$.

The performance improvement of MRC receiver over the number of diversity branches can be observed in Fig.3 and Fig.4. Compared to the no diversity case, performance improvement through MRC diversity is significant. Therefore MRC combining is a promising technique to overcome the inherent deteriorating performance of the energy detector at low SNR region. However, to obtain the $P_{d}$ of about 0.9 which is demanded by the first cognitive radio standard [12], even at low $\bar{\gamma}$ values, the performance improvement through diversity

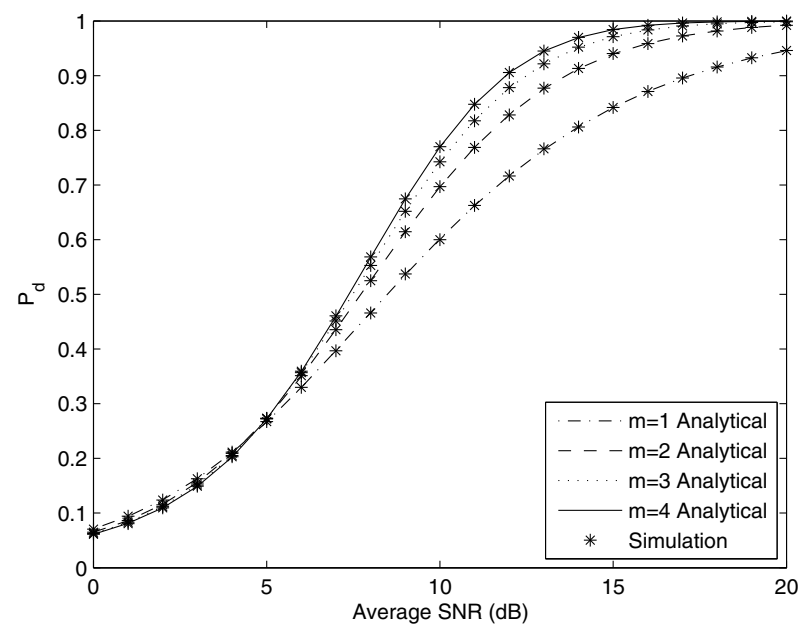

Fig. 1. Analytical and Simulation $\bar{P}_{d, N a k}$ for $u=2, m=\{1,2,3,4\}$ and $P_{f}=0.01$

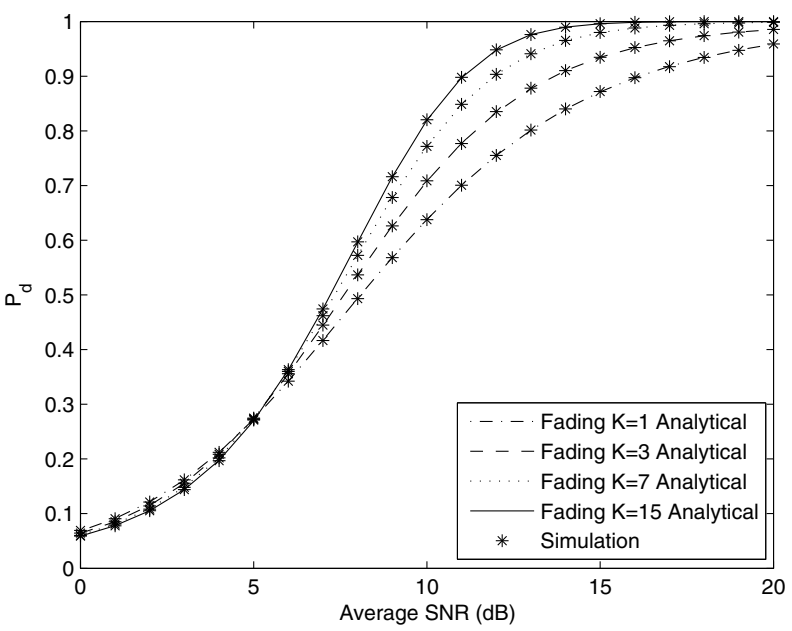

Fig. 2. Analytical and Simulation $\bar{P}_{d, R i c}$ for $u=2, K=\{1,3,7,15\}$ and $P_{f}=0.01$

gain alone is inadequate and thus other means need to be employed.

Making use of the integer constraint of $L m$ in (26), the performance is analyzed over non-integer values of $m$. The receiver performance over $\frac{1}{2}$ and $\frac{1}{3}$ multiples of $m$ values, respectively over dual and triple branch combining schemes is analyzed and some selected curves are shown in Fig.5. Due

$$
\begin{array}{r}
\bar{P}_{d, m r, R i c}=e^{-\frac{\lambda}{2}}\left(\frac{(1+K) \theta e^{-K \theta}}{\bar{\gamma}}\right)^{L} \sum_{n=1}^{\infty} \frac{a^{n-1}}{(n-1) !}\left(\frac{1}{(u-L-1) !} \lim _{z \rightarrow 0} \frac{d^{u-L-1}}{d z^{u-L-1}}\left[\frac{e^{\frac{\lambda z}{2}}}{(1-z)(z-\theta)^{L+n-1}}\right]\right. \\
\left.+\frac{1}{(L+n-2) !} \lim _{z \rightarrow \theta} \frac{d^{L+n-2}}{d z^{L+n-2}}\left[\frac{e^{\frac{\lambda z}{2}}}{z^{u-L}(1-z)}\right]\right)
\end{array}
$$




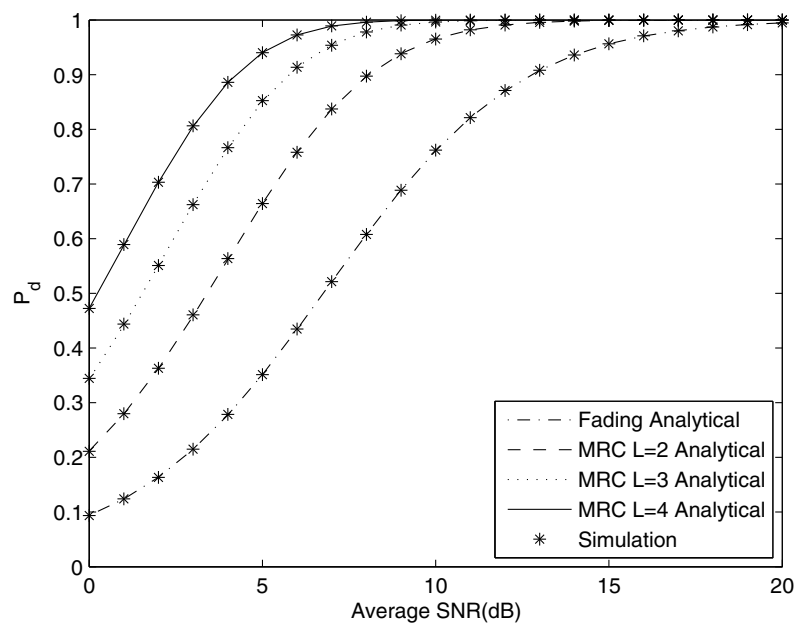

Fig. 3. Analytical and Simulation $\bar{P}_{d, m r, N a k}$ for $L=\{2,3,4\}, u=1$, $m=2$ and $P_{f}=0.01$

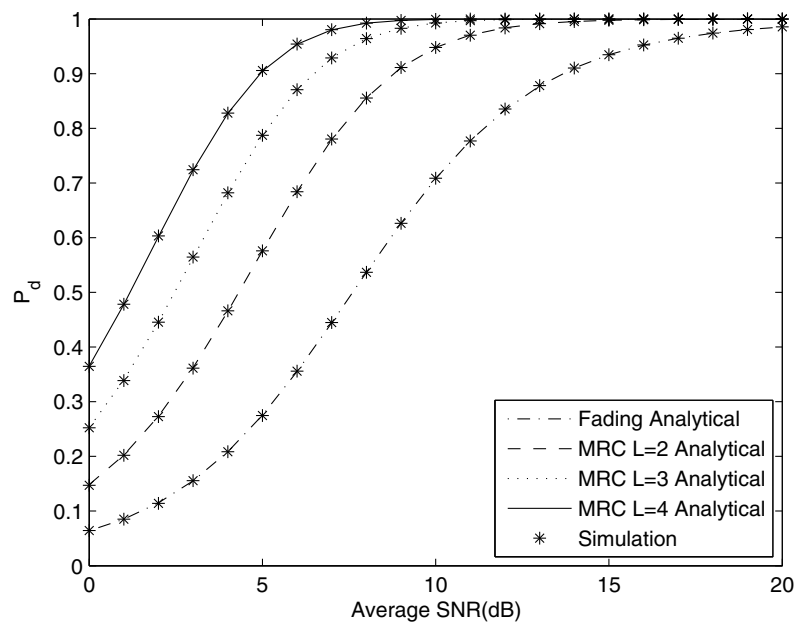

Fig. 4. Analytical and Simulation $\bar{P}_{d, m r, \text { Ric }}$ for $L=\{2,3,4\}, u=2$, $K=3$ and $P_{f}=0.01$

to the space limitations, detector performance over $u$ and high $P_{f}$ requirements is not shown.

\section{CONCLUSION}

The problem of detecting an unknown deterministic signal by using an energy detector has been considered. Applying a new approach based on the MGF of SNR and the contour integral representation of Marcum-Q function, the detector performance was evaluated. To the best of our knowledge, this approach has not previously been applied in analyzing the performance of an energy detector. Alternative results for detection probability over Nakagami- $m$ and Rician fading channels were derived. A new form of decision variable construction over a maximal ratio combiner was presented. Using this formulation, closed-form expressions for the average detection and false alarm probabilities over a MRC combined

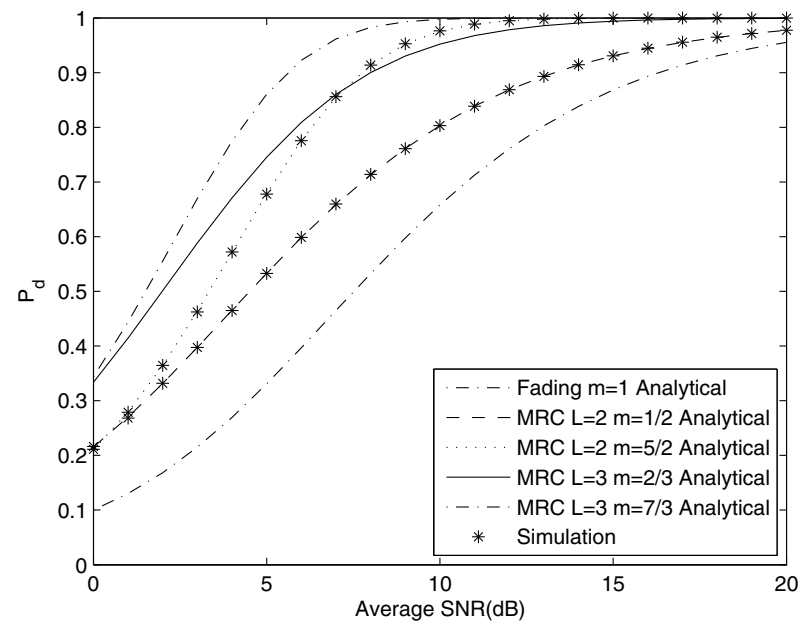

Fig. 5. Analytical and Simulation $\bar{P}_{d, m r, N a k}$ for $L=\{2,3\}, u=1$, $m=\left\{\frac{1}{2}, \frac{5}{2}, \frac{2}{3}, \frac{7}{3}\right\}$ and $P_{f}=0.01$

energy detector were derived. Numerical and simulation results were provided to support the proposed MGF based method, theoretical formulations and derivations. These results could readily be used in deciding the energy threshold value to be set to maintain a specified false alarm rate and the number of diversity branches to be used in detecting the spectrum holes by using an energy detector in a cognitive radio environment.

\section{REFERENCES}

[1] H. Urkowitz, "Energy detection of unknown deterministic signals," Proc. IEEE, vol. 55, no. 4, pp. 523-531, 1967.

[2] V. Kostylev, "Energy detection of a signal with random amplitude," in Proc. IEEE International Conference on Communications ICC 'O2, vol. 3, 2002, pp. 1606-1610.

[3] F. F. Digham, M.-S. Alouini, and M. K. Simon, "On the energy detection of unknown signals over fading channels," in Proc. IEEE International Conference on Communications ICC '03, vol. 5, 2003, pp. 3575-3579.

[4] _ , "On the energy detection of unknown signals over fading channels," IEEE Trans. Commun., vol. 55, no. 1, pp. 21-24, Jan. 2007.

[5] A. Pandharipande and J.-P. Linnartz, "Performance analysis of primary user detection in a multiple antenna cognitive radio," in Proc. IEEE International Conference on Communications ICC '07, 2007, pp. 64826486.

[6] A. H. Nuttall, "Some integrals involving the $Q$ function," in Naval Underwater Systems Center (NUSC) technical report, April, 1972.

[7] _ _ "Some integrals involving the $Q_{M}$ function," in Naval Underwater Systems Center (NUSC) technical report, May, 1974.

[8] S. P. Herath and N. Rajatheva, "Analysis of equal gain combining in energy detection for cognitive radio over Nakagami channels," in Proc. IEEE Global Telecommunications Conference IEEE GLOBECOM '08, 2008, pp. 1-5.

[9] —- "Analysis of diversity combining in energy detection for cognitive radio over Nakagami channels," in IEEE International Conference on Communications ICC '09, June, 2009, to appear.

[10] C. Tellamura, A. Annamalai, and V. Bhargava, "Contour integral representation for generalized marcum-q function and its application to unified analysis of dual-branch selection diversity over correlated Nakagami-m fading channels," in Proc. IEEE 51st VTC 2000-Spring Tokyo Vehicular Technology, vol. 2, 2000, pp. 1031-1034 vol.2.

[11] G. L. Stüber, Principles of mobile communication (2nd ed.). Norwell, MA, USA: Kluwer Academic Publishers, 2001.

[12] K. Cordeiro, C. Challapali and D. Birru, "IEEE 802.22: The first cognitive radio wireless regional area network standard," Communications Magazine, IEEE, pp. 130-138, Jan. 2009. 